\title{
Why Implement Distributed Systems in Municipal Music Schools in Colombia?
}

\author{
Leidy D. Ariza ${ }^{1} \&$ Carlos R. Orjuela ${ }^{1}$ \\ ${ }^{1}$ Technological faculty, Francisco Jose of Caldas District University, Bogota, Colombia \\ Correspondence: Leidy D. Ariza, Technological faculty, Francisco Jose of Caldas District University, Bogota, \\ Career 159 \# 136 -25, Colombia. Tel: 11-57-313- 3114595. E-mail: leidydi.ariza@gmail.com
}

Received: August 3, 2017

Accepted: August 16, 2017

Online Published: August 24, 2017

doi:10.5539/mas.v11n9p92

URL: https://doi.org/10.5539/mas.v11n9p92

\begin{abstract}
In Colombia, since 2003, the public policy "National Plan of Music for citizen Coexistence" has been implemented as a government measure, which provides music courses in each one of the country's municipalities. This plan does not take into account the use of technologies to share the experiences of each one of the schools.

Taking into account the above- mentioned points, this article focuses its attention on the search of technologies that can be used to share multimedia content such as Content Delivery Network (CDN), Learning Management System (LCMS) and Distributed Systems in order to indicate which technology is the most appropriate to fulfill this purpose.

In that sense, through the Wireshark tool, network traffic is captured for each one of the tests performed: Upload, display and deletion of videos for each configured technology (CDN, LCMS and Distributed Systems), having as comparison parameters the following aspects: Real-time Traffic, Total Traffic Vs Packet Loss, Communication Exchange and Protocol Hierarchies. After doing that, we proceed to take statistics to be analyzed and obtain the comparative results that are needed for this research.

Finally, one can conclude the comparison of the results of each technology: that it is appropriate that the municipal schools of music use distributed systems because the size of the packets sent is smaller than that one that is sent by CDN and CML technologies. There are no multiple communication jumps. No prior approval is required to publish the content and there is no limitation on the size of the content to be published.
\end{abstract}

Keywords: content delivery network, communication, distributed system, e-learning, learning content management system, multimedia, package, performance, traffic

\section{Introduction}

\subsection{Introduce the Problem}

1) Why is this problem important?

In Colombia, the connection in opportunities with access for the exercise of education and musical practice in conditions of equity and under the criteria that allows broad regional coverage has been limited. The Ministry of Culture through the "National Plan of Music for Coexistence", a public policy for music since 2003, seeks to strengthen and guarantee the population their right to know, practice and enjoy musical creation. In this way, it fosters the training and consolidation of Municipal Schools of Music in all the municipalities of the country, promoting musical education for children, young people and adults.

At present, there are 943 Municipal Music Schools created through an administrative act, corresponding to $73 \%$ of national coverage, with an approximate service of 93,000 children, adolescents and young people. Currently, these schools do not have a system to store and share such information, since all multimedia material is handled locally.

2) How does the study relate to previous work in the area? If other aspects of this study have been reported previously, how does this report differ from, and build on, the earlier report?

In order to carry out this article it was necessary to consult on different theses that have been interested in the matter to be addressed in this project. During the search of these theses, it was identified that some theses focused their attention on one of the technologies to share multimedia such as LMS, making comparative charts 
that allowed identifying between different platforms which one was the most appropriate for a certain institution. Another study focused its attention on the measurement of parameters to verify that LMS tool will allow adding new components to the platform and that its integration is acceptable.

Analysis of Productivity to Add Components in LMS Tools Applied to the Development of a Module Oriented to the New Management of Espoch Ratings.

This thesis is carried out in Riobamba, Ecuador in 2013. In this research, the authors pretend to select, under the measurement of parameters, the LMS tools that best suit the requirement to add new components. Once the tools have been selected, it will proceed to validate similarities of the characteristics and behavior that each one has, in order to be able to select the tool that best suits to the need. (Villacres \& Silva, 2013)

Comparative research of LCMS platforms used by universities at the national level.

This thesis is carried out in Machala in 2016. It shows a comparative study of the LCMS platforms used by ten universities of Ecuador that were selected for this research; it analyzes the functionalities, advantages and disadvantages of each virtual platform. Finally, a performance test of the virtual educational platform is performed, by using a computer tool that measures the behavior of the hardware along with the software, in terms of processing speed. (Armijos, 2016)

\section{State of the Art Construction of Technologies Used in Virtual Education}

This project is carried out in Pereira in 2009, this project that is related to the technologies that are used in virtual education, aims at providing all the necessary information for the research group Nyquist of Technological University of Pereira, to build a new platform for virtual education. This research tries to reveal aspects such as: traffic generated, bandwidth used, application capabilities, in order to determine the hardware requirements and the impact that the applications generate in the traffic level of the networks. (Arevalo, 2009)

3) What are the primary and secondary hypotheses and objectives of the study, and what, if any, are the links to theory?

\section{General Objective}

To query on the characteristics of the main technologies that allow to share multimedia contents and to establish through a comparative test the performance, speed of each technology and the cost - benefit relation in order to point out which one is suitable for Municipal Schools of Music.

\section{Specific Objectives}

To identify the characteristics and benefits of LMS, LCMS, CDN technologies and Distributed Systems.

To carry out the analysis of traffic network and the behavior of each one of the technologies, taking into account the selected comparative parameters.

To determine which one of the researched technologies best suits to the needs of the Municipal Schools of Music according to the results obtained.

\subsection{Explore Importance of the Problem}

This project analyzes the current situation regarding the local storage of information, the limited internet access in departments with presence of armed conflict actors and the centralized access to the multimedia production of the Municipal Music Schools in Colombia, as well as the lack of a system that allows to carry out the academic follow-up of the students, especially for those who, due to their condition of vulnerable population, are forced to change their physical location and want to continue their musical academic training in another municipal school near their new place of residence.

This research also includes a description of the most common alternatives to solve the problems that were previously mentioned such as: CDN technologies, LMS and Distributed Systems including its advantages, disadvantages, performance for both loading and unloading, visualization of files and ease of use for both educators and students.

\subsection{Describe Relevant Scholarship}

\subsubsection{Learning Management System (LMS)}

The e-learning platform, virtual campus or Learning Management System (LMS) enables distance learning through a virtual space. LMS facilitates distributed and collaborative learning from activities and content already developed, synchronously or asynchronously, using internet communication services such as mail, forums, videoconferences and chat. (Lopez, 2005) 
Within the Educational Software, there are free platforms that allow sharing information. We will mention the following ones:

\section{Learning Content Management System (LCMS)}

LCMS is defined as a web-based system that is used to create, approve, publish, manage and store educational resources. This system can be used by the designers who assemble the course, teachers who use the content to prepare their classes and students can also access the platform so as to strengthen their knowledge.

The content is stored within an LCMS as identifiable objects within containers or repositories which can be used independently and are available in an open way, meaning that anyone can access to the information that is needed.

One of the products is ATutor, distributed as a free software and compatible with e-learning standards for the export and import of content with other platforms and also with Learning Object Repositories. (Lopez, 2005)

\subsubsection{ATUTOR}

ATutor is a Web-based Learning Management System (LMS) created in open source software that is used to develop and deliver online courses. Educators can quickly assemble, package and redistribute Web-based educational content, easily import prepackaged content, and take their courses online.

ATutor's main features are: Accessibility, working groups, forums, create and share documents, store files, share storage space, assign blogs to working groups using mathematical notation in latex templates and multimedia objects when necessary for blog posts, content viewer, and a Concurrent Version System (CVS) to ensure the tracking of the documents worked and the historical archive as well.

\subsubsection{ACONTENT}

AContent is a learning content management system (LCMS) with an open-source software that is used to create interactive, accessible, and adaptable web-based learning content. It can be used in along with learning management systems to develop, share and store learning materials. AContent has the development of different kind of content, test authentication, and it also offers the opportunity to get the all the interoperability features in terms of content that ATutor has, producing a stand-alone tool that can be used with any system that supports IMS taking into account content interoperability standards.

Table 1. Advantages and Disadvantages of LCMS

\begin{tabular}{ll}
\hline Advantages & Disadvantages \\
\hline $\begin{array}{l}\text { Asynchronous and Synchronous Communication } \\
\text { Collaborative Groups }\end{array}$ & $\begin{array}{l}\text { Internet connection is required } \\
\text { The deployment of information depends on speed and } \\
\text { browsers } \\
\text { To publish information, it is necessary to have space } \\
\text { on storage server and previous approval by the } \\
\text { administrators of the platform for the final publication } \\
\text { of the course. }\end{array}$ \\
$\begin{array}{ll}\text { It reduces editing time by reusing templates and } \\
\text { learning objects }\end{array}$ & \\
Faster content delivery &
\end{tabular}

\subsubsection{Content Delivery Network CDN}

It is a network made up of a main server and several alternatives between which the content is kept updated to be delivered to the end users. These types of networks are usually private and corporative; nevertheless, they have recently been opened to the general public by offering content hosting according to the needs of the customers.

\section{Advantages:}

High availability of the content to offer, even with the unavailability of one or more of the substitutes.

Low latency and increased response time between substitutes and clients.

Control of content integrity preventing modification or elimination. 


\section{Disadvantages:}

High maintenance cost per year depending on the type of regional or global coverage.

\subsubsection{Distributed Systems (S.D)}

A Distributed System is a set of independent computers connected through a network, and with specific software that are shown to users as a single computer. This definition can be taken, taking into account the definitions given by Tanenbaum and Colouris.

Tanenbaum: A distributed system is a collection of independent computers that appear to the users of the system as a single computer. (Tanenbaum, 1996)

Colouris: A distributed system consists of a collection of autonomous computers, linked by a computer network and equipped with specific software. (Colouris, 1999)

\section{Characteristics:}

Distributed systems look for goals to achieve, systems to be faster, more efficient, more reliable, more accurate and more adaptable.

To provide greater quality of service and speed by increasing the number of servers.

To ensure operational continuity of the system, in which the most important aspect is not speed but availability.

To look for accuracy or computation ability, taking the background that the system delays in delivering the results. (Menéndez, 2009)

\section{Methodology}

The methodology will be divided into three sections: loading videos, video viewing and deleting videos, within each one finds the process to obtain the results, which consists in capturing the frames with the program Wireshark from entry to the platform (FTP, HTTPS or NFS), analysis of the traces and generation of comparing graphs.

This methodology will be used in the tests for each of the technologies: CDN, LCMS and distributed systems. The parameters that were chosen to make the comparison of each one of the technologies are: real-time traffic, generation of packets per second identifying the quantity of packets sent (Tx packets), received (Rx packets), original sizes of files and total sizes in the frames, total vs. lost packet traffic ratio, communication exchange capability, and protocol hierarchy.

\subsection{Capture and Traffic Analysis}

To perform a network traffic analysis, it is required to identify all the necessary data to make the performance comparison of the different technologies that we are going to test. For this test, we will use the tool WIRESHARK 2.2.7 For the traffic capture process, the following steps were followed:

\subsubsection{To capture traffic using CDN Technology}

A CDN must be configured to allow sending the video or the multimedia content, when uploading videos via FTP (FileZilla Client), the Wireshark program is activated allowing real-time traffic capture. It is important to obtain the .pck file that Wireshark generates in order to get the required data for the traffic analysis, get the display of each one of the videos that were uploaded from the platform.

After following all the previous steps, the corresponding data to the selected parameters were obtained.

And finally, the results are retained and then compared with the results obtained by the other technologies

\subsubsection{To Capture Traffic Using LCMS Technology}

For doing that, Docebo platform is selected for this test.registration on the platform is done with a corporate email (in this case, a student mail account from District University). Nevertheless, open emails such as Hotmail, Gmail, Yahoo, etc. are not allowed. Once you enter on the platform, a folder is created to store the multimedia content. The platform allows you to upload 1 video at a time while activating the Wireshark program that allows you to capture network traffic in real time. It's important to obtain the .Pck file that generates Wireshark in order to get the required data for the traffic analysis, get the display of each one of the videos that were uploaded from the platform. After following all the previous steps, the data corresponding to the selected parameters were obtained; the results are retained and then compared with the results obtained by the other technologies. 


\subsubsection{To Capture Traffic Using Distributed Systems}

\section{Considerations:}

Inner shared folder of the school network with access only to domain users with permissions to write, read and modify content. The Website exposure with Directory browsing permission (in read mode to prevent accidental or incidental deletion or modification of the content) from the server at the external network level through the WIFI of the municipal school or the WIFI network of the Municipal Mayor's Office. For this reason, login processes, advertising banners, and platform load timeout are not present in this analysis. The upload protocol is NFS which allows you to copy content within the same network from one computer to another, copied from an USB device, a pen drive, a video camera, a photo camera or just a mobile phone. Displaying videos from the published intranet site through the WIFI network of the school or the Municipal Mayor's Office. It's mandatory to obtain the .Pck file that generates Wireshark in order to get the required data for the traffic analysis by following all the previous steps, the corresponding data to the selected parameters were obtained and the result were retained and then compared with the results obtained by the other technologies.

\subsection{Statistics Collected from Tests with CDN Technology}

The first thing that done was the loading of videos, and as a result, the following graphs were obtained:

Generation of packages:

\begin{tabular}{|c|c|c|c|c|c|c|c|c|c|c|}
\hline \multicolumn{9}{|c|}{ I Wireshark - Endpoints - Carga_de_videos_CDN } & $\square$ & \multirow[t]{2}{*}{$\times$} \\
\hline IPv $4 \cdot 43$ & $\mathrm{TCP} \cdot 124$ & & & & & & & & & \\
\hline \multicolumn{2}{|l|}{ Address } & Packets & Bytes & Tx Packets & Tx Bytes & Rx Packets & Rx Bytes & Latitude & Longitude & $\hat{\imath}$ \\
\hline \multirow{3}{*}{\multicolumn{2}{|c|}{$\begin{array}{l}\text { derhabmann } \\
\text { push-24.cdn77.com } \\
40.97 .124 .34 \\
\end{array}$}} & 106.861 & $101 \mathrm{M}$ & 44.906 & $37 \mathrm{M}$ & 61.955 & $64 \mathrm{M}$ & - & - & \\
\hline & & 37.465 & $34 \mathrm{M}$ & 15.302 & $922 \mathrm{k}$ & 22.163 & $33 \mathrm{M}$ & - & - & \\
\hline & & 27.211 & $28 \mathrm{M}$ & 19.815 & $27 \mathrm{M}$ & 7.396 & $416 k$ & - & - & $\checkmark$ \\
\hline \multirow{2}{*}{\multicolumn{2}{|c|}{$\square$ Name resolution }} & \multirow{2}{*}{\multicolumn{3}{|c|}{$\square$ Limit to display filter }} & & & & & \multicolumn{2}{|c|}{ Endpoint Types - } \\
\hline & & & & & Copy & 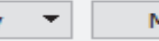 & Map & Close & Help & \\
\hline
\end{tabular}

Figure 1. Ipv4

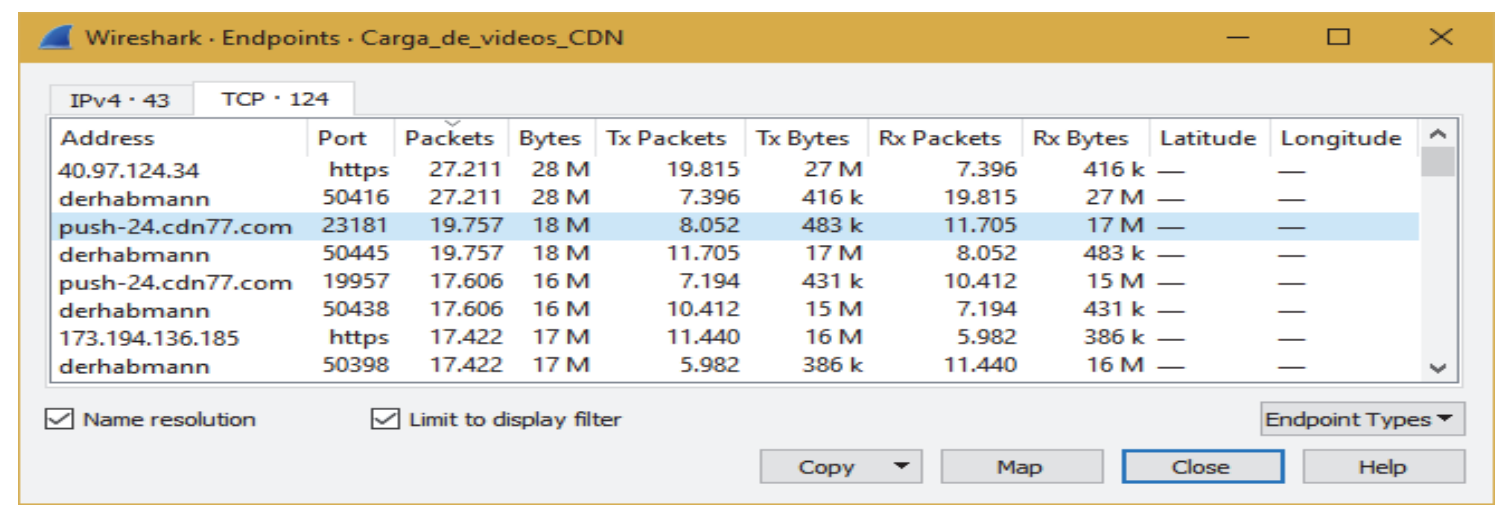

Figure 2. Tcp 


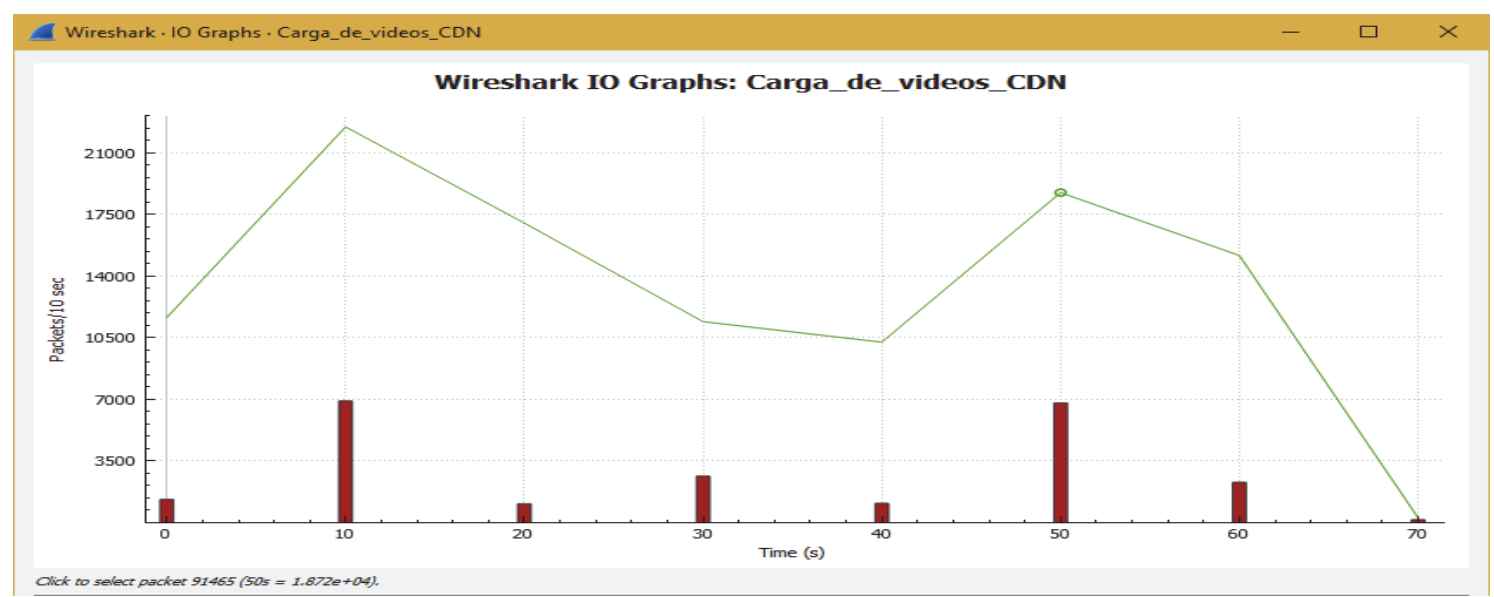

Figure 3. Total Traffic vs. Packet Loss Ratio

$\triangle$ Wireshark · Conversations : Carga_de_videos_CDN

\begin{tabular}{|c|c|c|c|c|c|c|c|c|c|c|c|}
\hline $\operatorname{IPv} 4 \cdot 41$ & & & & & & & & & & & \\
\hline Address A & Address B & Packets & Bytes & Packets $A \rightarrow B$ & Bytes $A \rightarrow B$ & Packets B $\rightarrow$ A & Bytes $B \rightarrow A$ & Abs Start & Duration & Bits $/ 5 A \rightarrow B$ & Bits $/ \mathrm{s} B \rightarrow \mathrm{A} \wedge$ \\
\hline push-24.cdn77.com & derhabmann & 37.465 & $34 \mathrm{M}$ & 15.302 & $922 k$ & 22.163 & $33 \mathrm{M}$ & $20: 59: 18.787732$ & 58.3938 & $126 k$ & $4545 k$ \\
\hline 40.97 .124 .34 & derhabmann & 27.211 & $28 \mathrm{M}$ & 19.815 & $27 \mathrm{M}$ & 7.396 & $416 k$ & $20: 59: 12.055311$ & 69.8496 & $3189 \mathrm{k}$ & $47 k$ \\
\hline 173.194 .136 .185 & derhabmann & 17.422 & $17 \mathrm{M}$ & 11.440 & $16 \mathrm{M}$ & 5.982 & $386 k$ & $20: 59: 13.653969$ & 68.4409 & $1976 \mathrm{k}$ & $45 \mathrm{k} v$ \\
\hline \multirow[t]{2}{*}{$\square$ Name resolution } & \multirow{2}{*}{\multicolumn{3}{|c|}{$\square$ Limit to display filter }} & \multirow{2}{*}{\multicolumn{3}{|c|}{$\square$ Absolute start time }} & \multicolumn{5}{|c|}{ Conversation Types } \\
\hline & & & & & & & Copy $-F$ & follow Stream... & Graph... & Close & Help \\
\hline
\end{tabular}

Figure 4. Package Exchanges (Conversation) - Ipv4

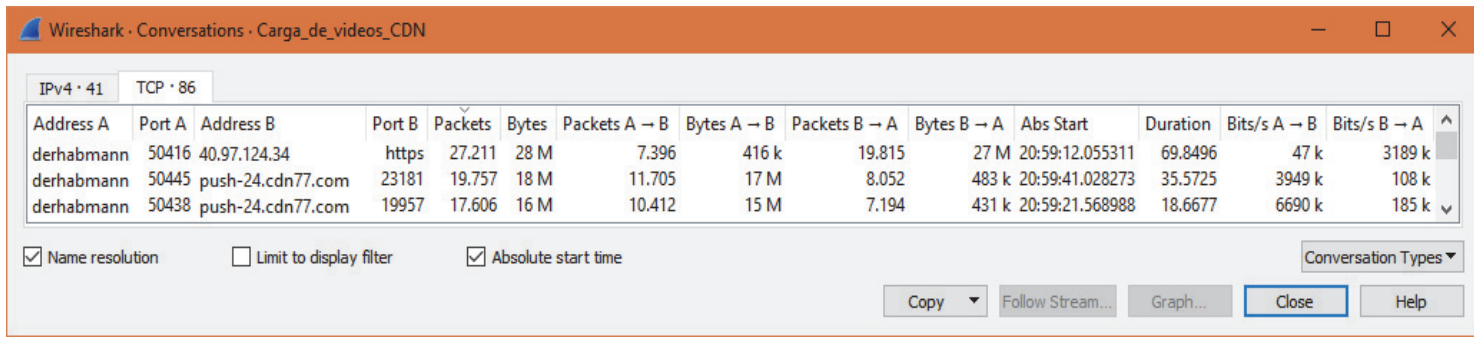

Figure 5. Package Exchanges (Conversation) - Tcp

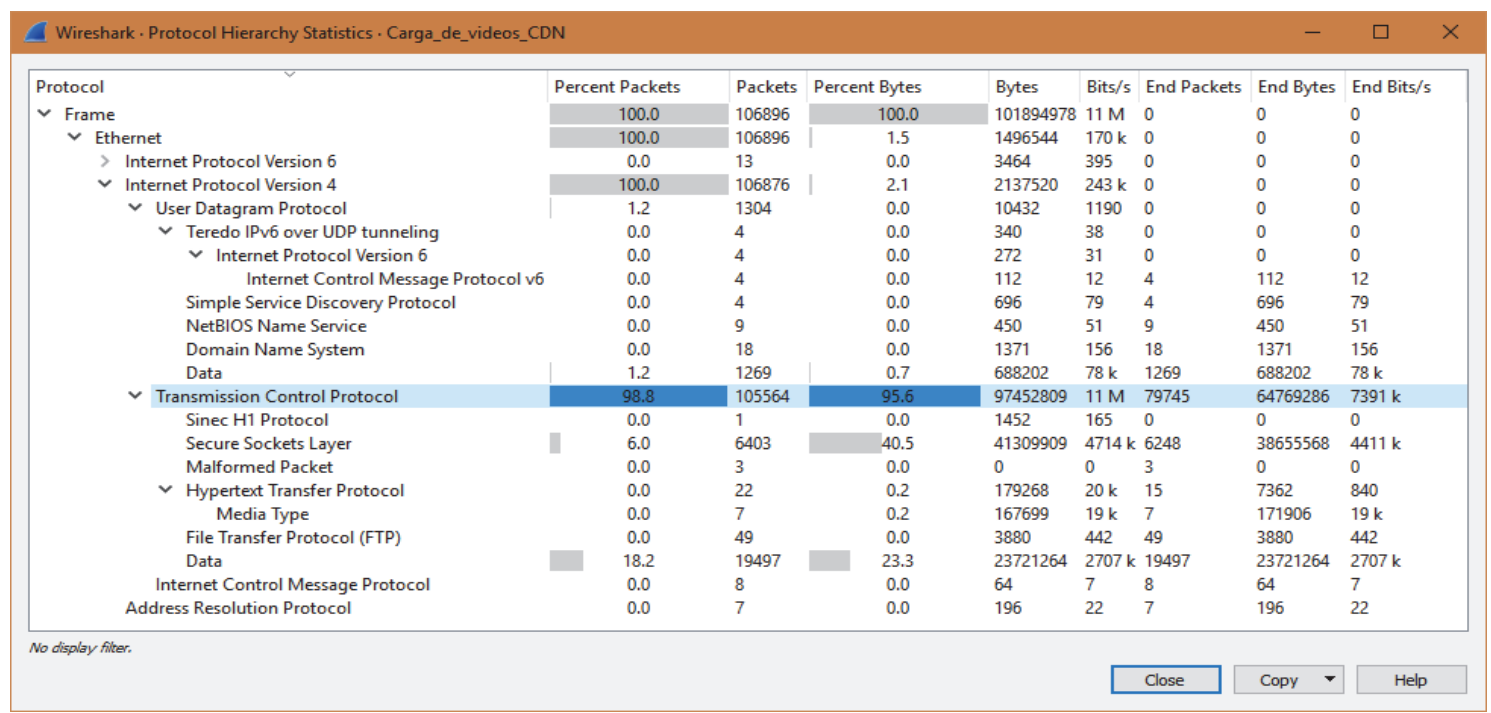

Figure 6. Protocol Hierarchy 
Below is the capture of network traffic at the time of display video 1 in $\mathrm{CDN}$

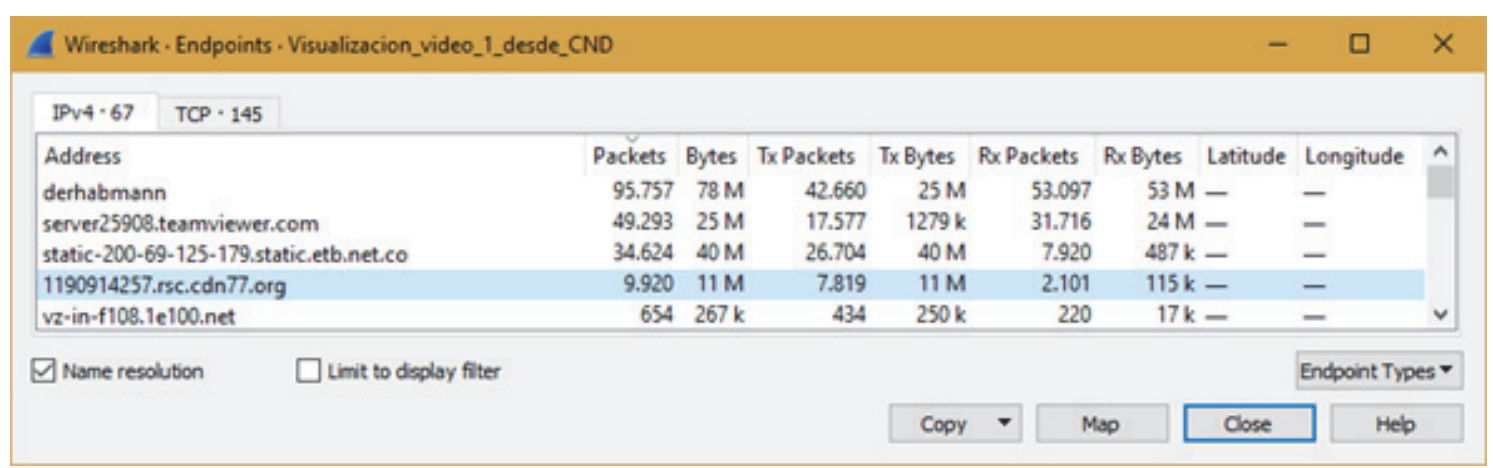

Figure 7. Network Traffic Ipv4

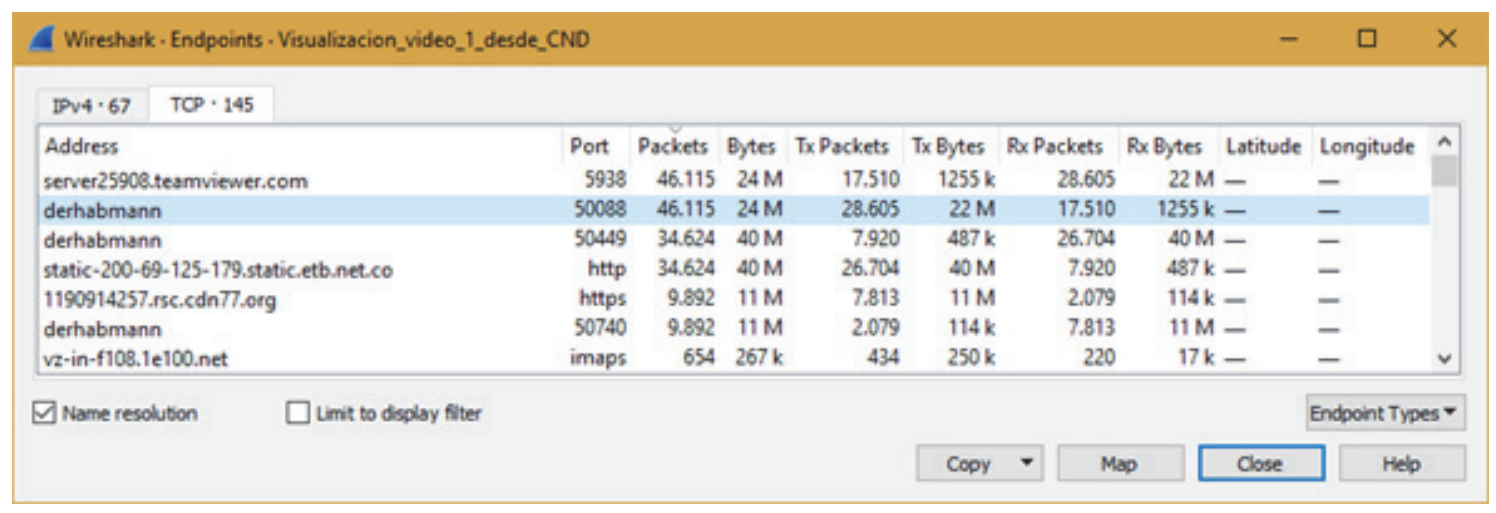

Figure 8. Network Traffic TCP

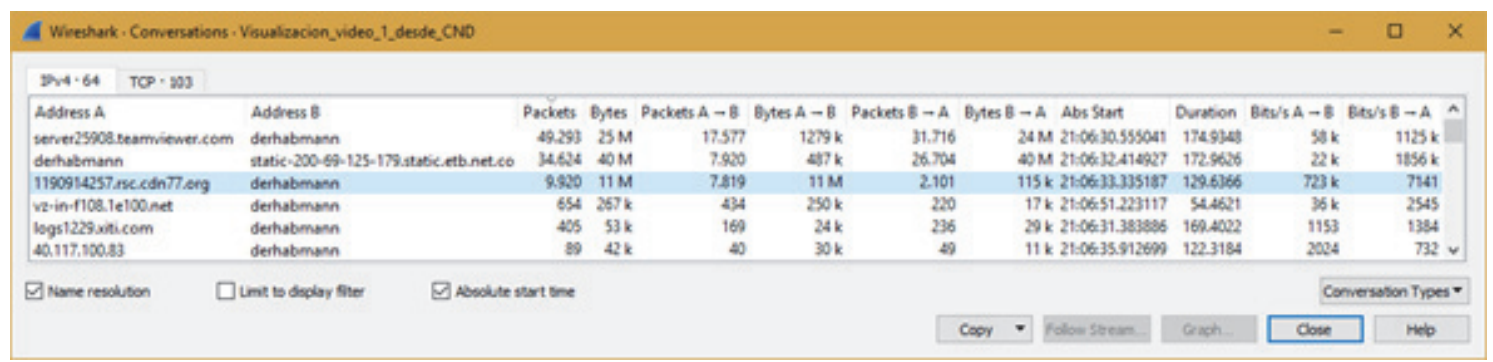

Figure 9. Package Exchanges (Conversation) - Ipv4

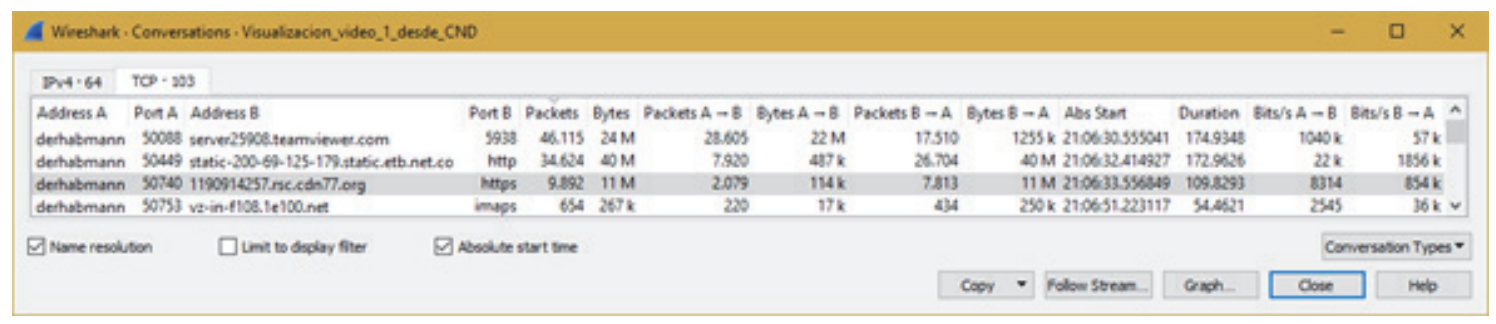

Figure 10. Package Exchanges (Conversation) - TCP

Below is the capture of network traffic at the time of display video 2 in LMS 


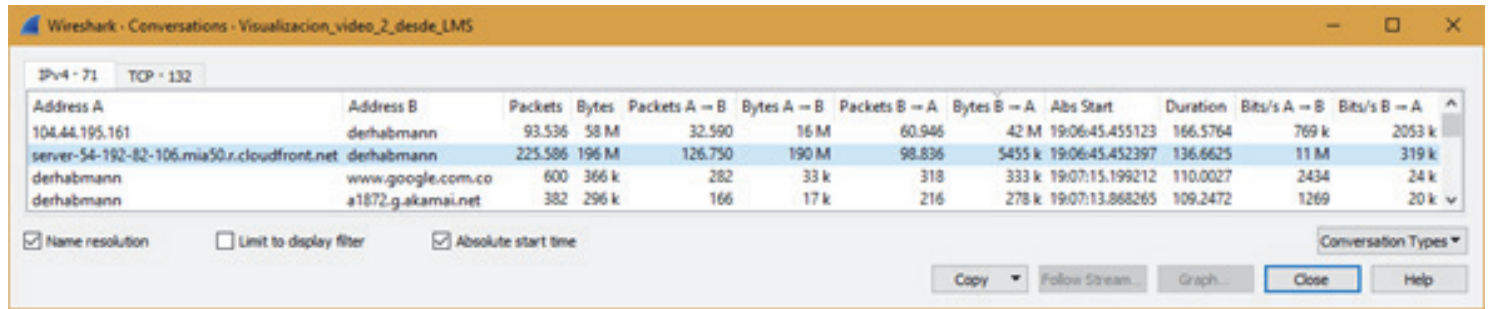

Figure 11. Network Traffic Ipv4

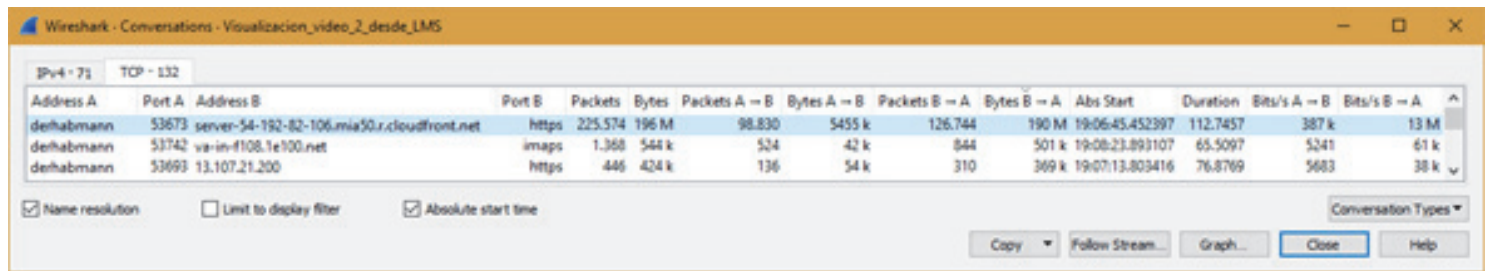

Figure 12. Network Traffic TCP

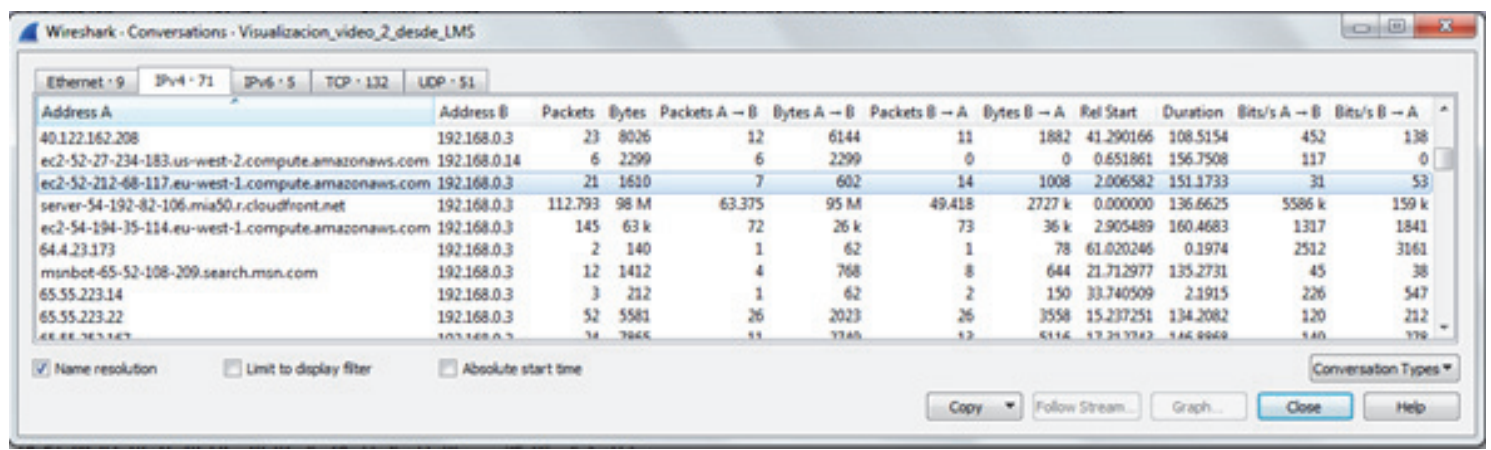

Figure 13. Package Exchanges (Conversation) - Ipv4

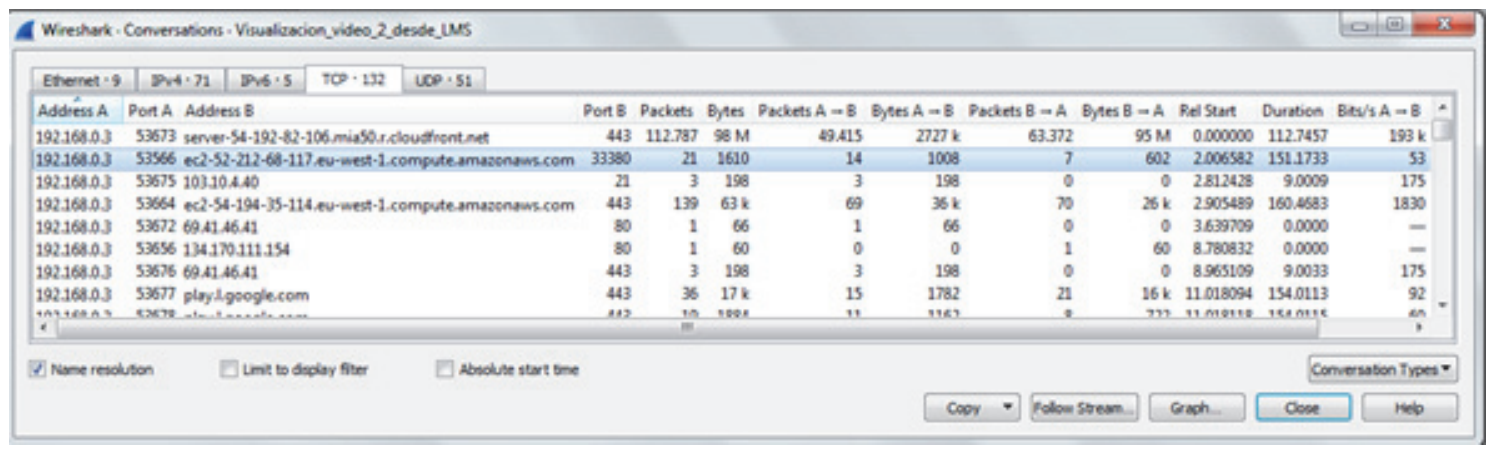

Figure 14. Package Exchanges (Conversation) - TCP

Below is the capture of network traffic at the time of display video 1 in Distributed system

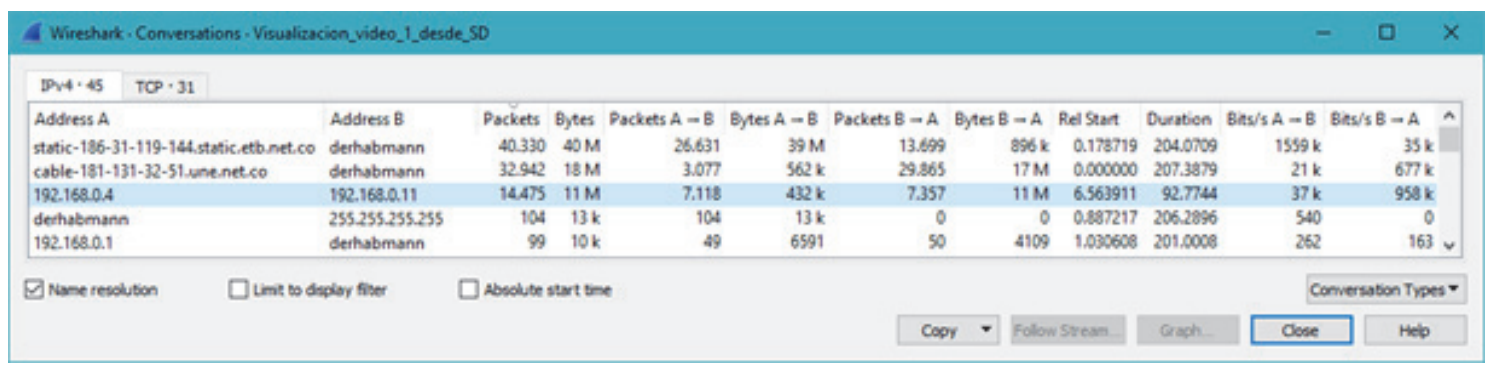


Figure 15. Network Traffic Ipv4

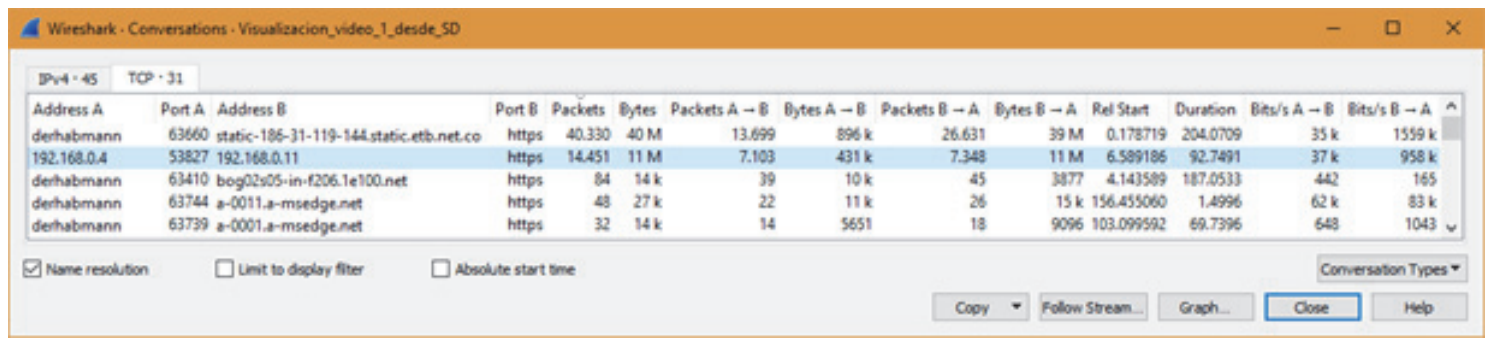

Figure 16. Network Traffic TCP

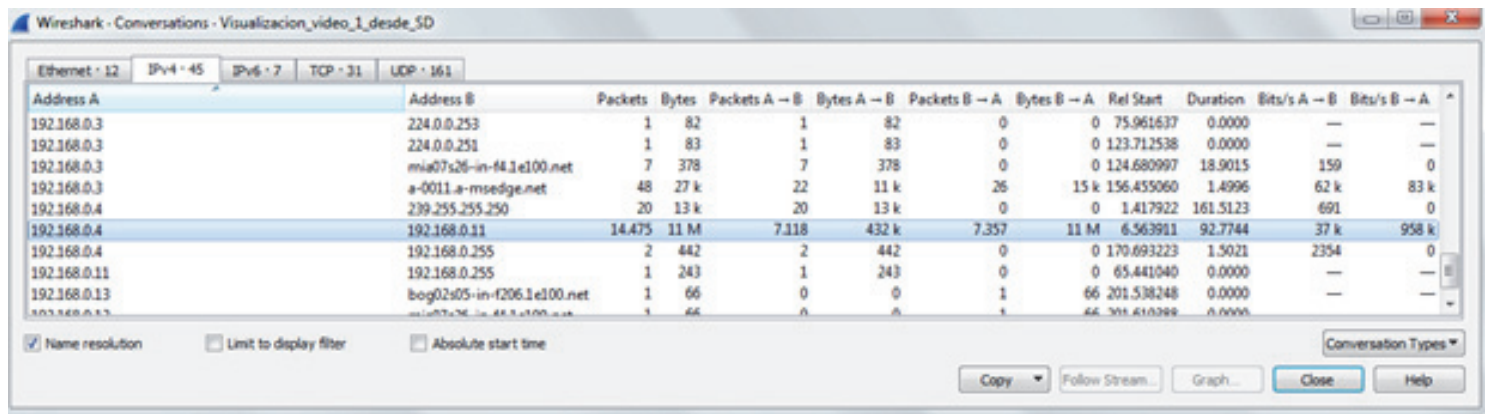

Figure 17. Package Exchanges (Conversation) - Ipv4

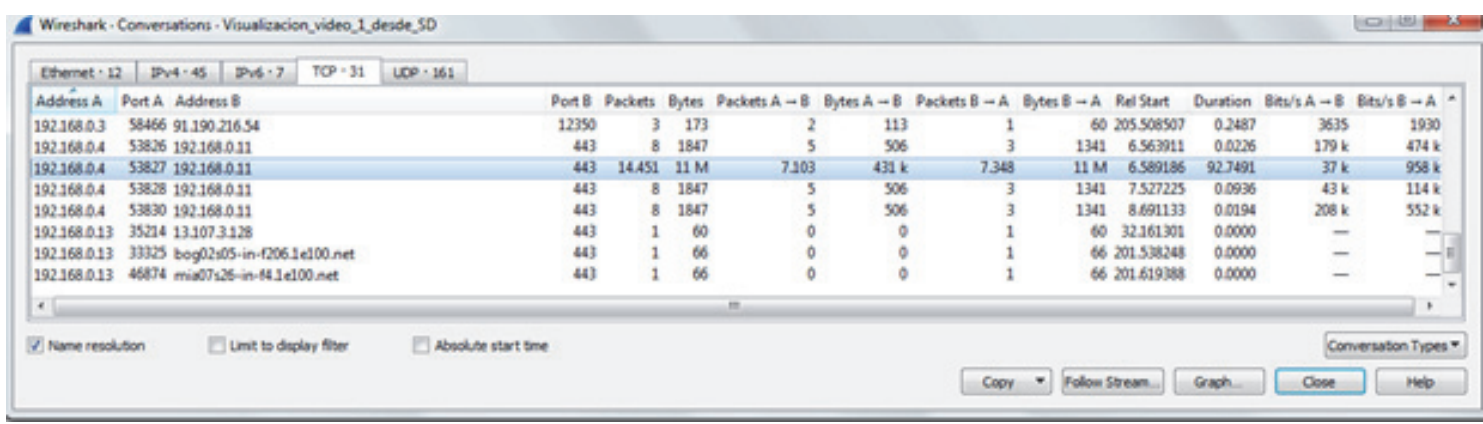

Figure 18. Package Exchanges (Conversation) - TCP

\section{Results and Discussion}

It is important to keep this table in mind for comparing technologies

Table 2. Names of the Videos

\begin{tabular}{ll}
\hline File & Name \\
\hline Video 1 & Playing_with_the_names.mp4 \\
Video 2 & Musical_Initiation_Class.mp4 \\
\hline
\end{tabular}

Table 3. Load of videos referring to each one of the technologies selected for this research.

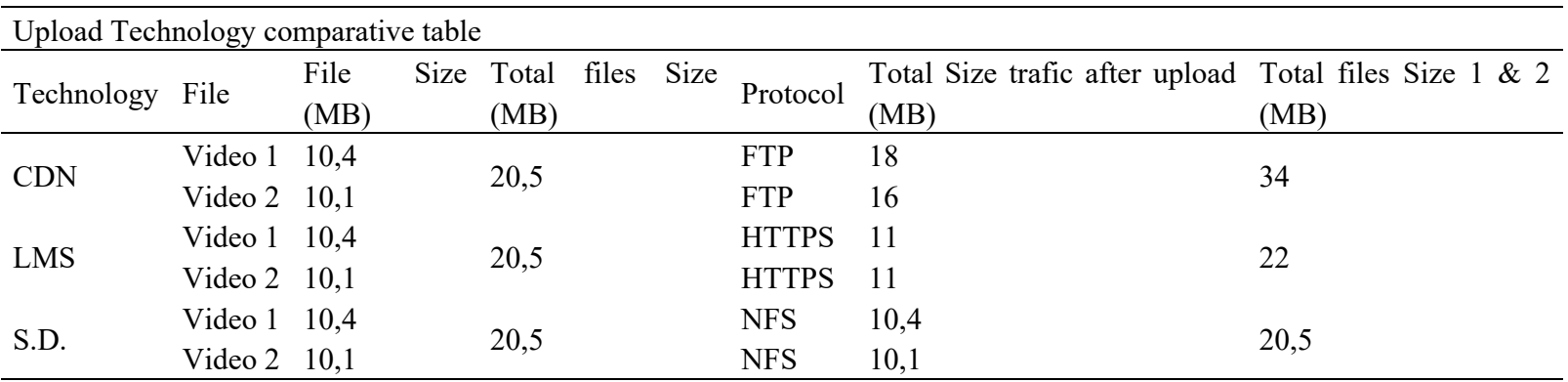


As a result of the first table there is an increase in the size of the traffic-level file on the CDN and LMS platforms, compared to the original size, except for the Distributed System (DS) in which as it is a local copy of the file to a repository (in this case to a folder of an exposed website with browsing permission in read- only mode) there is not any increase.

Table 4. Visualization Technology comparative table

\begin{tabular}{|c|c|c|c|c|c|c|}
\hline Technology & File & $\begin{array}{l}\begin{array}{l}\text { File } \\
\text { Size } \\
(\mathrm{MB})\end{array} \\
\end{array}$ & $\begin{array}{l}\text { Total files Size } \\
\text { (MB) }\end{array}$ & Protocol & $\begin{array}{l}\text { Total Size incoming } \\
\text { trafic (MB) }\end{array}$ & $\begin{array}{l}\text { Total files Size } 1 \& \\
2(\mathrm{MB})\end{array}$ \\
\hline \multirow{2}{*}{$\mathrm{CDN}$} & Video 1 & 10,4 & \multirow{2}{*}{20,5} & HTTPS / 2 & 11 & \multirow{2}{*}{25} \\
\hline & Video 2 & 10,1 & & HTTPS /2 & 14 & \\
\hline \multirow{2}{*}{ LMS } & Video 1 & 10,4 & \multirow{2}{*}{20,5} & HTTPS & 11 & \multirow{2}{*}{24} \\
\hline & Video 2 & 10,1 & & HTTPS & 13 & \\
\hline \multirow{2}{*}{ S.D. } & Video 1 & 10,4 & \multirow{2}{*}{20,5} & HTTPS & 11 & \multirow{2}{*}{22} \\
\hline & Video 2 & 10,1 & & HTTPS & 11 & \\
\hline
\end{tabular}

On the other hand, as a result of the second table, there is an increase in the size of the file at the traffic level in each one of the platforms, showing a marked growth in both CDN and LMS. In the case of CDN, it is due to jumps in the communication between hosts until arriving at the nearest server (Brazil) with respect to the place where the measurement has been made (Colombia). For the case of LMS, it makes a jump between different hosts (login site, Amazon CDN and Finally Google video services). In the case of DS, the communication is at the local level of the network since the URL https: //croserver/Media / is exposed from the Web server in whose Media folder the videos are located and which can also be entered without authentication, avoiding jumps in the communication between the client device (Laptop, Smartphone, Tablet, etc.).

Table 5. Costs / Details Technology comparative table

\begin{tabular}{llllll}
\hline Technology & Plans & Capacity GB & Cost Per month (US\$) & Maximum Files & Maximum active users \\
\hline \multirow{2}{*}{ CDN } & Min & 6000 & 230 & \multirow{2}{*}{ According to capacity } & unrestricted \\
& Max & 100000 & 1890 & & 50 \\
& Min & Unrestricted & 230 & Unlimited & 600 \\
LMS & Max & Unrestricted & 350 & & 50 \\
& Min & 1000 & $\sim 100$ & According to capacity & 480 \\
S.D. & Max & 7000 & $\sim 200$ &
\end{tabular}

As a result of the third table, we have that at cost / benefit level both CDN and LMS have high costs associated with the availability of content storage capacity and the number of simultaneous users (concurrence). On the contrary, the D.S is characterized by sharing the resources of the server, workstation or desktop computer selected for this service.

For each one of the technologies, it was chosen the one that is best adapted, taking into account different aspects such as the ease of use both for the administrator by the municipal school and for the end user, including ease of publication; visualization, access to each one of the sites, both for the CDN and the LMS for being high availability services. Test time was taken into account for free in order to test the functionalities, in this case a maximum period of 14 days.

In the case of the CDN, CDN77 was selected which offered the file upload service both for web and for FTP. In this case, FTP was used because it is required fewer steps for connection, creation of folders and the corresponding upload of the video files.

For the LMS case, it was selected Docebo for ease of use against Atutor which required complete publication of the material for reviewing and prior approval by the platform administrators for their final publication, to allow uploading any type of file without restriction of a specific size (for free account maximum 50 GB of storage), compared to one of the platforms that is most often used at the educational level (Moodle) which does not allow loading of multimedia files and has a maximum restriction of $10 \mathrm{MB}$ per file.

For the Distributed System, it was selected a virtualized server with a virtual PC that has the following characteristics: Windows 2012 r2 with 8GB RAM, 80GB disk storage with 2-core processor AMD FX 8350 to 
4GHZ, private network connection shared with the hosts equipment with IIS 8.5 with the internal TESTSD website to a folder called MEDIA where videos are hosted, posted with security HTTPS and whose address was (https: // croserver / Media /) and private IP address 192.168.0.11, static from the internet connection modem.

The website has directory browsing permission in read mode that allows viewing and / or downloading the videos but not delete or modify them. In respect of the estimated prices, it was made on the quote of a workstation (Hewlett Packard Z800) with 2 TB of storage and 3 years guarantee.

For the cases of test for the display of the videos in the Distributed System, it was used a Smartphone and an additional laptop of the hosting equipment, thus confirming the operability and facility of access to the information which is a premise of any distributed system.

The choice of a local Distributed System was made based on the 3 tables that were mentioned in the results and additionally taking into account that the municipal school budget is largely focused on the acquisition and maintenance of musical instruments. Moreover, the facility of publication of multimedia content once the website is created and the inner side of the school, the Media folder can be presented as a shared resource with permissions of writing to a group of authorized users to upload or delete the published videos doing even easier maintenance of the website without the intervention of technical staff for that purpose.

\section{References}

AContent. No date. Retrieved from http://www.atutor.ca/acontent/

Akamai. (2017). Teach talk. Retrieved from https://www.akamai.com/

Arevalo, M. G. (2009). Construction of the state of art of the technologies used in virtual education. (Undergraduate thesis). Retrieved from http://repositorio.utp.edu.co/dspace/bitstream/handle/11059/1336/371334A683.pdf?sequence=1

Ariza, R. L., \& Silva, N. Y. (2013). Web Information System for Management of administrative processes for schools Municipal Sustainable Music in the Cundinamarca Department. Tekhne Journal District University, 10(1). https://doi.org/10.13140/RG.2.2.13828.22406

Armijos, S. N. (2016). Study comparative of the LCMS platforms used by universities at the national level. (Undergraduate thesis). Retrieved from http://repositorio.utmachala.edu.ec/bitstream/48000/7564/1/TCUAIC_2016_ISIST_CD0005.pdf

ATutor Features. No date. Retrieved from http://www.atutor.ca/atutor/features.php

Berger, A., Weaver, N., \& Beverly, R. (2013). Internet Nameserver IPv4 and IPv6 address Relationships. (pag. 91-104). New York, NY: Association for Computing Machinery (ACM). DOI:10.1145/2504730.2504745

Cacheda, F., Formoso, V., \& Carneiro V. (2007). Analysis of performance of distributed systems of recovered of information on the web., 5(6). Retrieved from http://www.ewh.ieee.org/reg/9/etrans/ieee/issues/vol05/vol5issue6Oct.2007/5TLA6_14Cacheda.pdf

Content Delivery Network. CDN77.com. (2017). Retrieved from https://www.cdn77.com/

Docebo. No date. Retrieved from https://www.docebo.com/es/

Enriquez, V. L. (2004, November 10). LCMS and learning objects. University digital Journal. Retrieved from http://moodle2.unid.edu.mx/dts_cursos_mdl/pos/ED/AV/AM/07/LMS.pdf

Levin, S., \& Schmidt, S. (2014). IPv4 to IPv6: Challenges, solutions and lessons. Telecommunications Policy, 38, 1069-1068. https://doi.org/10.1016/j.telpol.2014.06.008

Martinez, P. S., Korobeinikov, A., \& Alcon P. O. (2012, May 20). Content Distribution Networks (CDN). Retrieved from https://www.youtube.com/watch?v=4VK60vQGukA

MinCultura. (2015). Retrieved from http://www.mincultura.gov.co/Paginas/default.aspx

Molina, V. R. (2015). Construction of concept of technology in a learning virtual network, 20(1). https://doi.org/10.14483/udistrital.jour.enunc.2015.1.a01

National consumer protection network. (2017). Retrieved from http://www.redconsumidor.gov.co/publicaciones/actividades_de_las_alcaldias_en_la_proteccion_al_consu midor_pub

Normas, A. P. A. (2017). Sixth edition. Retrieved from http://normasapa.net/2017-edicion-6/

Ovalle, A. D., \& Jimenez, A. J. (2006). Intelligent environment distributed of learning: Integration of ITS y 
CSCL through pedagogical agents. (Num. 6, pp. 89-104). Retrieved from http://repository.eia.edu.co/bitstream/11190/571/1/REI00051.pdf

Padilla, A. J., Paradells, A. J., \& Becerra, S. L. (2011). A proposal to improve the bandwidth in the radio channel in HMIPV6 Networks (HMIPV6-BI), 9(5). https://doi.org/10.1109/TLA.2011.6030966

Razzak, A. H. A., Handa, S., \& Ramana, M. M. (Jun de 2014). Providing the Secure Data Transmission in the Network Using Open Source Packet Analyzer. International Journal of Computer trends and Technology (IJCTT), 12(1). https://doi.org/10.14445/22312803/IJCTT-V12P103

Villacres, V., \& Silva, P. (2013). Productivity Analysis to add components in the LMS Tools applied to the developed of one module oriented at the new management of qualifications of the Espoch. (Undergraduate thesis). Recovered from http://dspace.espoch.edu.ec/bitstream/123456789/2717/1/18T00534.pdf

\section{Copyrights}

Copyright for this article is retained by the author(s), with first publication rights granted to the journal.

This is an open-access article distributed under the terms and conditions of the Creative Commons Attribution license (http://creativecommons.org/licenses/by/4.0/). 